\title{
Age-related changes in two- and three-dimensional morphology of type-identified endplates in the rat diaphragm
}

\author{
Toshimi Suzuki $\cdot$ Akio Maruyama \\ Takao Sugiura · Shuichi Machida · Hirofumi Miyata
}

Received: 20 August 2008/Accepted: 31 October 2008/Published online: 30 November 2008

(C) The Physiological Society of Japan and Springer 2008

\begin{abstract}
To examine the age-related morphological changes in the motor endplate of type-identified muscle fibers, 20 male Wister rats were divided into 2-month-, 10-month-, 24-month- and 30-month-old groups ( $n=5$ in each group). Three segments of mid-costal diaphragm muscle were removed, and then a fluorescent doublelabeling technique was used to visualize the endplates on type-identified muscle fibers. Endplates were labeled with $\alpha$-bungarotoxin-tetramethylrhodamine. Muscle segments were first incubated in antibodies to MHC isoforms (I and/ or IIa) and then labeled by a second antibody with FITC. The endplates were imaged using 3D confocal microscopy with two lasers. In each age group, the planar area and volume of endplates on type-IIx/b muscle fibers were larger than those on type-I and -IIa muscle fibers, while the normalized planar areas of the endplate (endplate area/ muscle fiber diameter) and the mean thickness of the endplate (volume/endplate area) were identical on all fiber types within the same age group. Decreased endplate density (endplate area/surrounding area) in the old diaphragm indicated fragmentation of the endplate, especially on type IIx/b fibers. These morphological changes may
\end{abstract}

T. Suzuki · A. Maruyama $\cdot$ H. Miyata $(\bowtie)$

Department of Biological Sciences,

Graduate School of Medicine, Yamaguchi University,

Yoshida 1677-1, Yamaguchi 753-8515, Japan

e-mail: hiro@yamaguchi-u.ac.jp

T. Sugiura

Department of Health and Sports Sciences,

Yamaguchi University, Yamaguchi, Japan

S. Machida

Department of Physical Recreation,

School of Physical Education, Tokai University,

Kanagawa, Japan lead to functional deficiency and selective denervation of type-IIx/b muscle fiber with aging.

Keywords Aging · Diaphragm · Endplate area . $3 \mathrm{D}$ analysis

\section{Introduction}

One of the most remarkable age-related changes in skeletal muscle is the selective degeneration of fast-twitch muscle fibers (type IIx/b) where the number and relative area of fast fibers decrease with the increase of slow-twitch fibers (type I) $[1,2]$. The underlying reason hypothesized for the alternation is that the selectively denervated fast-twitch muscle fibers are again innervated by slow-type motoneurons, a phenomenon known as axonal sprouting that results in an increased number of hybrid fibers and aggregation of slow-twitch muscle fibers. These muscle fiber changes with aging concomitantly result in an altered skeletal muscle contractile property [3-5]. The cycle of denervation and subsequent reinnervation of type IIx/b fibers could possibly be initiated at the neuromuscular junction where there is a gap between the presynaptic nerve terminal and postsynaptic endplate [6]. In the previous studies, the age-related deterioration process of endplates at the neuromuscular junction was evaluated, and the results revealed that the endplates become hollow, structurally wider in shape and eventually atrophy with age [7-9]. Furthermore, Prakash and Sieck [10] have demonstrated that, by using a confocal microscopic technique, more significant expansions of the neuromuscular junction of type IIx and IIb fibers were found in the diaphragm muscle of 24-month-old rats, as compared to those of 6-month-old rats. However, two timepoints observation (6- and 24-month-old) is not enough to 
distinguish between the development and aging-related morphological changes in the endplate.

The purpose of this experiment was to examine the effects of development and aging on endplate morphological changes in type-identified muscle fibers of the rat diaphragm. To investigate the type-specific endplate morphology, rat diaphragm muscles of four different age groups were used. Each muscle fiber type was identified by classification based on myosin heavy-chain isoform expression in muscle fibers. Moreover, to perform precise endplate morphological observation, the endplates on the muscle fibers were reconstructed in three- and twodimensional images with a confocal laser microscope.

\section{Methods}

\section{Animal treatment}

Experiments were performed on 20 male Wistar rats aged 2 months $(n=5,272 \pm 30 \mathrm{~g}), 10$ months $(n=5,518 \pm$ $35 \mathrm{~g}), 24$ months $(n=5,626 \pm 92 \mathrm{~g})$ and 30 months $(n=5,548 \pm 48 \mathrm{~g})$. The animals were anesthetized by intraperitoneal (IP) injection of pentobarbital sodium $(60 \mathrm{mg} / \mathrm{kg})$, and the diaphragm muscle was excised. Muscle segments of $5 \mathrm{~mm}$ wide were sectioned in a parallel direction with the muscle fiber from the mid-costal region of the left diaphragm muscle.

All experimental and animal care procedures were approved by the Committee on Animal Care and Use in Yamaguchi University and followed the American Physiological Society Animal Care Guidelines.

Endplate image and muscle fiber typing

The muscle segments were washed and immersion fixed in $2 \%$ paraformaldehyde. The fixed samples were blocked for nonspecific staining by using $10 \%$ donkey serum in $0.1 \mathrm{M}$ Tris-buffered saline containing $1 \%$ Triton. The tissue was washed and incubated in $5 \mu \mathrm{g} / \mathrm{ml} \alpha$-bungarotoxin-tetramethylrhodamine (SIGMA, St. Louis, MO) for $3 \mathrm{~h}$. Then each muscle segment was incubated overnight in solutions with an antibody to myosin heavy-chain (MHC) isoform I (BA-D5), an antibody to MHC isoform IIa (SC-71) or a mixture of two antibodies (BA-D5 and SC-71). Then the segments were incubated in a solution with a second antibody with FITC for $6 \mathrm{~h}$. At least 25 endplates on typeidentified muscle fibers in each segment were imaged using a laser-scanning confocal system (C1, Nikon, Tokyo) mounted on a microscope (E600, Nikon, Tokyo) and equipped with a HeNe-Green laser (543 nm for rhodamine and $488 \mathrm{~nm}$ for FITC). First, several images of optical sections at $2.4-\mu \mathrm{m}$ steps were obtained with low magnification $(20 \times$ dry object lens, Nikon, Tokyo), and then images of type-identified endplate were obtained at $1.0-\mu \mathrm{m}$ steps with high magnification $(60 \times$ oil-immersion objective lens, Nikon, Tokyo). In total, more than 125 images in each group were analyzed. Sets of optical sections were transferred to a comprehensive imagemanipulation and -analysis software package (3D-Doctor, Solution System, Tokyo). Two-dimensional (2D) stack images for each labeled endplate were reconstructed (Fig. 1), and the endplate areas, surrounding area and endplate density (endplate area/surrounding area $\times 100$ ) were measured. In addition, the muscle fiber diameter was measured to calculate the normalized endplate area (endplate area/muscle fiber diameter). Although the fiber diameter is variable depending on the confocal plane, the largest diameter on 2D stack images that was adjacent to the endplate was measured. Three-dimensional (3D) images for each labeled endplate were reconstructed (Fig. 2), and the volume, surface area and thickness of the endplate (volume/2D area of the endplate) were measured.
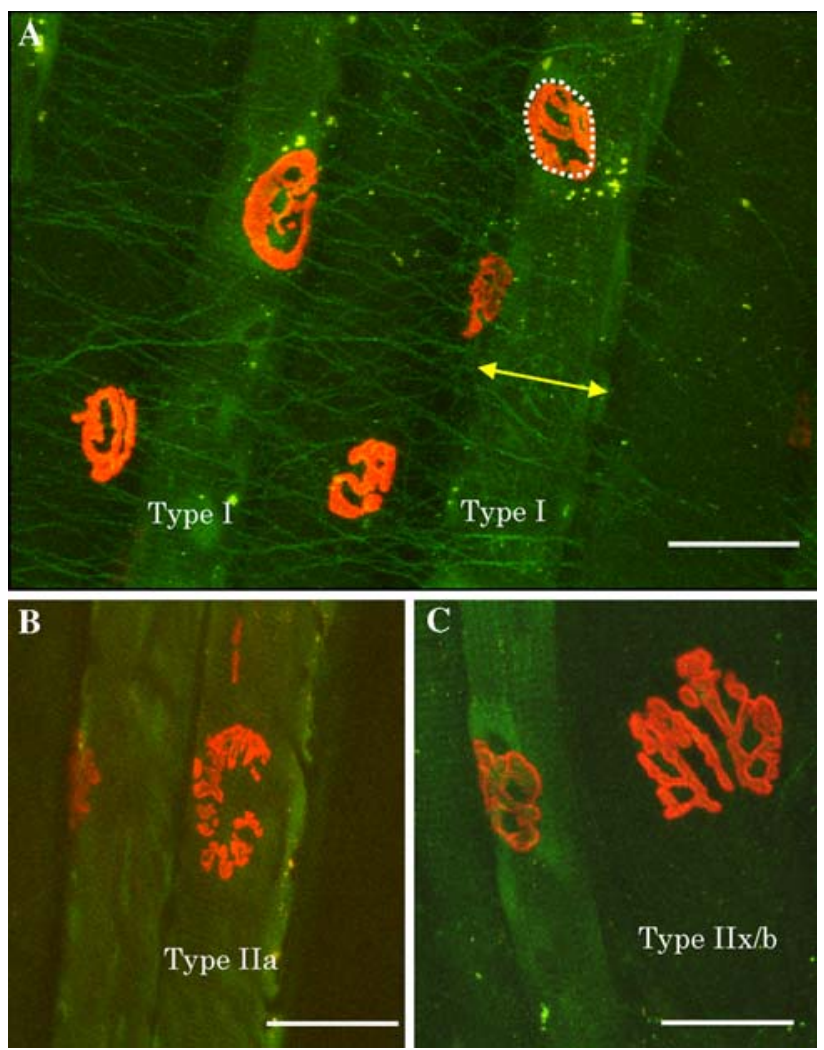

Fig. 1 Two-dimensional images of labeled endplates (red) on typeidentified muscle fibers with anti-myosin heavy chain (green) from 24-month-old rat. The acetylcholine receptor area was stained in red, and the surrounding area was determined as the outer border of the acetylcholine receptor area marked with white dashed line (a). Muscle diameter is indicated by a yellow arrow (a). These data were used to calculate the endplate normalized area and endplate surrounding area. White bar indicates $30 \mu \mathrm{m}$ 
Fig. 2 Typical threedimensional images of endplate on each fiber type from 2-month-old (a, c, e) and 30-month-old (b, d, e) rats. Using software (3D-Doctor), the three-dimensional endplates were reconstructed, and endplate volume, endplate surface area and mean thickness (volume/2D area of endplate) were calculated. White bar indicates $10 \mu \mathrm{m}$

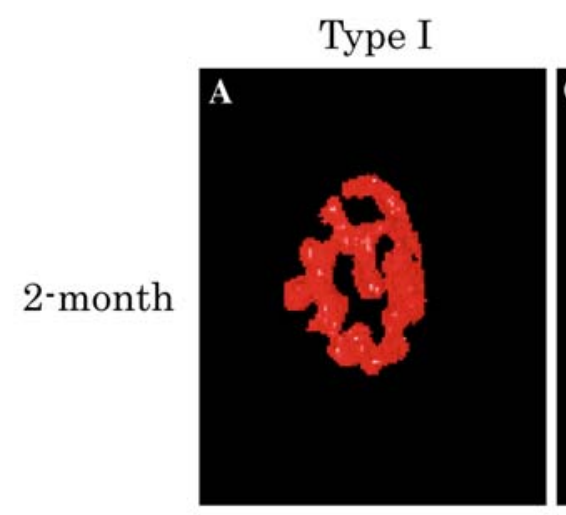

Type IIa
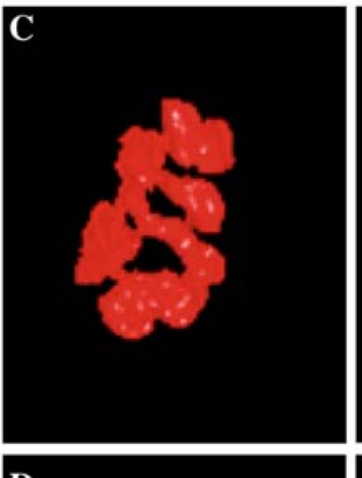
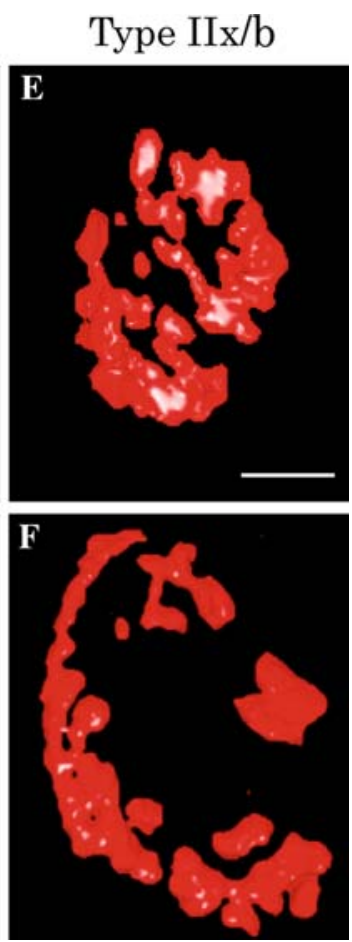

\section{Statistics}

The results obtained in this study were analyzed by twoway ANOVA with age groups (2-, 10-, 24- and 30-monthold) and fiber types (type I, IIa and IIx/b) as grouping variables. Post-hoc analysis was performed using a $t$-test with the Bonferroni adjustment method. In all cases, statistical significance was set at $P<0.05$. All values are reported as the mean \pm standard deviation.

\section{Results}

Two-dimensional morphology of endplates

According to the MHC isoform expression, muscle fiber type was classified as type I (positive fiber for BA-D5), type IIa (positive fiber for SC-71) or type IIx/b (negative fiber for both BA-D5 and SC-71). The fiber diameter was smallest in type I fibers and largest in IIx/b fibers in all age groups (Table 1). There was no age-related difference in the diameter in type I and type IIa fibers. However, type $\mathrm{IIx} / \mathrm{b}$ diameter in the 30-month-old group was significantly larger than those in the 2-month-old group.

The endplate area was smallest in type I fiber and largest in type IIx/b fibers in all age groups (Table 1). In type IIx/b fibers, the endplate area in the 10-, 24- and 30-month-old groups was significantly larger than those in the 2-month- old group, and the endplate normalized area for the muscle diameter in the 10-, 24- and 30-month-old groups was significantly larger than those in the 2-month-old group.

The endplate density, calculated by the endplate area divided by the endplate surrounding area $\times 100(\%)$, was highest in the 2-month group and followed in rank order by $10-<24-<30$-month groups in all fiber types (Table 1). In type IIx/b, the endplate density in the 30-month group was significantly lower than those in the 2-month group.

Three-dimensional morphology of the endplate

The endplate volume and surface area on each muscle fiber type are summarized in Table 2 . The endplate volume and surface area were largest in type IIx/b and smallest in type I fiber in all age groups. In type IIx/b fibers, the endplate volume and surface area were significantly larger in the 30-month group than those in the 2-month group.

The endplate thickness (volume/2D area of endplate) was identical in all fiber types, and no age-related changes were found.

\section{Discussion}

The purpose of this experiment was to evaluate developmental and age-related morphological change in endplates on type-identified muscle fibers. In type IIx/b muscle fibers, 
Table 1 Effect of aging on two-dimensional muscle fiber and endplate structures
Values are means \pm standard deviation

${ }^{a}$ Significant difference $(P<0.05)$ vs. type I

${ }^{b}$ Significant difference $(P<0.05)$ vs. 2 -month-old group

Table 2 Effect of aging on three-dimensional endplate structures

\begin{tabular}{|c|c|c|c|c|}
\hline \multirow[t]{2}{*}{$2-\mathrm{D}$} & \multirow[t]{2}{*}{ Age (month) } & \multicolumn{3}{|c|}{ Muscle fiber type } \\
\hline & & I & IIa & $\mathrm{IIx} / \mathrm{b}$ \\
\hline \multirow[t]{4}{*}{ Endplate area $\left(\mu \mathrm{m}^{2}\right)$} & 2 & $238 \pm 62$ & $314 \pm 64$ & $384 \pm 77^{\mathrm{a}}$ \\
\hline & 10 & $378 \pm 90$ & $496 \pm 130$ & $790 \pm 145^{\mathrm{a}, \mathrm{b}}$ \\
\hline & 24 & $327 \pm 94$ & $457 \pm 122$ & $741 \pm 218^{\mathrm{a}, \mathrm{b}}$ \\
\hline & 30 & $284 \pm 83$ & $434 \pm 160$ & $780 \pm 240^{\mathrm{b}}$ \\
\hline \multirow[t]{4}{*}{ Endplate surrounding area $\left(\mu \mathrm{m}^{2}\right)$} & 2 & $321 \pm 95$ & $439 \pm 115$ & $543 \pm 132^{\mathrm{a}}$ \\
\hline & 10 & $588 \pm 195$ & $800 \pm 300$ & $1,435 \pm 358^{\mathrm{a}, \mathrm{b}}$ \\
\hline & 24 & $529 \pm 157$ & $784 \pm 234$ & $1,344 \pm 411^{\mathrm{a}, \mathrm{b}}$ \\
\hline & 30 & $462 \pm 142$ & $779 \pm 281$ & $1,475 \pm 493^{\mathrm{a}, \mathrm{b}}$ \\
\hline \multirow[t]{4}{*}{ Endplate density (\%) } & 2 & $74.1 \pm 7.7$ & $71.5 \pm 7.5$ & $70.7 \pm 8.5$ \\
\hline & 10 & $64.3 \pm 11.0$ & $62.0 \pm 11.8$ & $55.1 \pm 5.6$ \\
\hline & 24 & $61.8 \pm 8.0$ & $58.3 \pm 7.3$ & $55.1 \pm 7.1$ \\
\hline & 30 & $61.5 \pm 7.5$ & $55.7 \pm 7.4$ & $52.9 \pm 5.1^{\mathrm{b}}$ \\
\hline \multirow[t]{4}{*}{ Muscle fiber diameter $(\mu \mathrm{m})$} & 2 & $21.1 \pm 2.9$ & $30.3 \pm 2.8^{\mathrm{a}}$ & $39.1 \pm 3.5^{\mathrm{a}}$ \\
\hline & 10 & $25.2 \pm 3.2$ & $34.5 \pm 3.1^{\mathrm{a}}$ & $51.3 \pm 7.4^{\mathrm{a}}$ \\
\hline & 24 & $23.4 \pm 3.2$ & $33.6 \pm 3.4^{\mathrm{a}}$ & $47.6 \pm 7.3^{\mathrm{a}}$ \\
\hline & 30 & $21.3 \pm 4.0$ & $33.2 \pm 3.3^{\mathrm{a}}$ & $51.3 \pm 6.5^{\mathrm{a}, \mathrm{b}}$ \\
\hline \multirow[t]{4}{*}{ Normalized endplate area $\left(\mu \mathrm{m}^{2} / \mu \mathrm{m}\right)$} & 2 & $11.3 \pm 2.6$ & $10.4 \pm 2.0$ & $9.8 \pm 1.7$ \\
\hline & 10 & $15.0 \pm 3.6$ & $14.4 \pm 4.7$ & $15.4 \pm 3.3^{\mathrm{b}}$ \\
\hline & 24 & $14.0 \pm 3.4$ & $13.6 \pm 3.0$ & $15.6 \pm 3.2^{\mathrm{b}}$ \\
\hline & 30 & $13.3 \pm 2.8$ & $13.1 \pm 3.3$ & $15.2 \pm 2.6^{\mathrm{b}}$ \\
\hline
\end{tabular}

\begin{tabular}{lrlll}
\hline 3-D & Age (month) & \multicolumn{3}{l}{ Muscle fiber type } \\
\cline { 3 - 5 } & & I & IIa & IIx/IIb \\
\hline Endplate volume $\left(\mu \mathrm{m}^{3}\right)$ & 2 & $1,610 \pm 633$ & $2,044 \pm 653$ & $2,877 \pm 1,097$ \\
& 10 & $2,503 \pm 1,251$ & $3,289 \pm 1,640$ & $4,961 \pm 1,348$ \\
& 24 & $2,793 \pm 1,252$ & $3,843 \pm 2,071$ & $6,217 \pm 2,813$ \\
& 30 & $1,892 \pm 938$ & $2,974 \pm 1,778$ & $5,054 \pm 1,938^{\mathrm{a}, \mathrm{b}}$ \\
Endplate surface area $\left(\mu \mathrm{m}^{2}\right)$ & 2 & $1,619 \pm 507$ & $2,137 \pm 617$ & $2,617 \pm 835$ \\
& 10 & $2,432 \pm 887$ & $3,069 \pm 1,208$ & $4,742 \pm 1,758$ \\
& 24 & $2,757 \pm 1,046$ & $3,836 \pm 1,683$ & $5,863 \pm 2,280$ \\
Endplate thickness $(\mu \mathrm{m})$ & 30 & $2,231 \pm 770$ & $3,675 \pm 1,726$ & $5,983 \pm 1,984^{\mathrm{a}, \mathrm{b}}$ \\
& 2 & $6.8 \pm 2.1$ & $6.5 \pm 2.1$ & $7.5 \pm 2.4$ \\
& 10 & $6.6 \pm 3.2$ & $6.6 \pm 2.8$ & $6.3 \pm 2.7$ \\
& 24 & $8.5 \pm 2.8$ & $8.4 \pm 3.3$ & $8.4 \pm 2.8$ \\
& 30 & $6.7 \pm 2.7$ & $6.9 \pm 2.9$ & $6.5 \pm 2.3$ \\
\hline
\end{tabular}

Values are means \pm standard deviation

a Significant difference $(P<0.05)$ vs. type I

b Significant difference $(P<0.05)$ vs. 2 -month-old group the endplate density (endplate area/surrounding area) was significantly smaller in the 30-month group than in the 2-month group. None of the parameters in type I and type IIa muscle fibers indicated significant developmental and age-related changes.

The typical age-related fiber atrophy was not observed, but rather fiber growth was observed, and the diameter of the 30-month group was significantly greater than the 2-month group. However, in type IIx/b muscle fibers of older diaphragm muscles, both the endplate receptors and surrounding area were larger compared to those in the 2-month group. Moreover, the normalized endplate area was also greater in size in older diaphragm muscle compared to that in the 2-month group. In a previous study, Prakash and Sieck [10] demonstrated a significant expansion in endplate area on type IIx and IIb fibers in rat diaphragm muscle with aging. These adaptations were appropriate to maintain the level of safety factor secured by increased Ach release and the number of Ach receptors accomplished by nerve terminal and endplate expansion. In our results, the endplate area 
was not only enlarged, but also became less dense in contrast to its surrounding area. The enlargement and subsequent scattering of the endplate area may be a result of compensation to the decreased Ach receptor sensitivity or affinity in the central portion of the endplate area. The underlying reason may be related to age-related change in the membrane cholesterol to phospholipid ratio, which changes the membrane fluidity known to be associated with acetylcholine receptor affinity $[11,12]$.

It is also possible that the expressions of basal laminar proteins, involved in maintaining the integrity of the endplate and its corresponding nerve terminal, e.g., the neural cell adhesion molecule, agrin [13], and neuregulin [14], are different across fiber types and thus account for differences in endplate fragmentation. It has been clearly demonstrated that expression of these basal laminar proteins is altered by conditions that affect the integrity of the endplate and nerve terminal [15-17]. These results were in accordance with the frequency of fiber type-specific recruitment patterns during normal respiration, in which only type I and type IIa motor units are recruited and type IIx/IIb motor units are rarely recruited in the diaphragm muscle [18]. Furthermore, it is of considerable interest that our results clearly indicate age-related endplate remodeling proceeded earlier in time compared to the muscle fiber remodeling. Although there were no significant age-related differences in the endplate density on type IIx/b muscle fibers, the endplate density of the 10- and 24-month groups was $22 \%$ smaller than the 2-month groups. These results suggested that age-related muscle fiber atrophy may follow the endplate deterioration with advancing age.

It is well known that the endplates have specialized architecture with a primary and secondary gutter to enlarge the surface area. Based on three-dimensional images, in a previous study using confocal microscopy, it was observed that the primary gutter depth of the endplate on type I fiber was greater than that on type II fiber, and this contributed to the greater endplate surface area on type I fibers [19]. Therefore, to discuss the morphological properties of the endplate with aging, three-dimensional evaluation on morphological properties of the endplates was also achieved in our study. The results revealed that three-dimensional analysis of the endplate also showed muscle fiber type-specific remodeling with aging. In type IIx/b fibers exclusively, increased surface area and volume were observed without any significant remodeling of those in type I and type IIa fibers. However, the endplate thickness was not different among all fiber types and age groups. The results suggested that the increased volume and the surface area of endplates were attributed to the expansion of the acetylcholine receptor expression area rather than the age-related changes in the endplates' fine architecture.
In conclusion, these results together suggested that a type-specific endplate enlargement and fragmentation begins as early as 10 months of age and occurs without muscle fiber atrophy with age. These endplate morphological changes may lead to the functional alteration of motor units and selective denervation on type IIx/b muscle fiber and subsequent fiber atrophy with age.

Acknowledgements This work was supported by a grant from The Japanese Ministry of Education, Science and Culture (no. 17300220).

\section{References}

1. Lexell J, Taylor CC, Sjöström M (1988) What is the cause of aging atrophy? Total number, size and proportion of different fiber types studied in whole vastus lateralis muscle from 15to 83-year-old men. J Neurol Sci 84:275-294. doi:10.1016/ 0022-510X(88)90132-3

2. Shono E, Yamano S, Miyata H (2005) Functional and metabolic adaptation of the rat diaphragm to aging and compensatory increased activity. Jpn J Phys Fitness Sports Med 54:121-131

3. Larsson L (1995) Motor units: remodeling in aged animals. J Gerontol A Biol Sci Med Sci 50:91-95

4. Rooms MR, Rice CL, Vandervoort AA (1997) Age-related changes in motor unit function. Muscle Nerve 20:679-690. doi:10.1002/(SICI)1097-4598(199706)20:6<679::AID-MUS4> 3.0.CO; $2-5$

5. Lexell J (1995) Human aging, muscle mass, and fiber type composition. J Gerontol A Biol Sci 50:11-16

6. Gutmann E, Hanzlíková V (1966) Motor unit in old age. Nature 209:921-922. doi:10.1038/209921b0

7. Robbins N, Fahim MA (1985) Progression of age changes in mature mouse motor nerve terminals and its relation to locomotor activity. J Neurocytol 14:1019-1036. doi:10.1007/BF01224810

8. Courtney J, Steinbach JH (1981) Age changes in neuromuscular junction morphology and acetylcholine receptor distribution on rat skeletal muscle fibers. J Physiol 320:435-447

9. Edström L, Larsson L (1987) Effects of age on contractile and enzyme-histochemical properties of fast- and slow-twitch single motor units in the rat. J Physiol 392:129-145

10. Prakash YS, Sieck GC (1998) Age-related remodeling of neuromuscular junctions on type-identified diaphragm fibers. Muscle Nerve 21:887-895. doi:10.1002/(SICI)1097-4598(199807)21:7< 887::AID-MUS6 $>3.0 . C O ; 2-2$

11. Yeagle PL (1985) Cholesterol and the cell membrane. Biochim Biophys Acta 822:267-287

12. Smith DO, Williams KD, Emmerling M (1990) Changes in acetylcholine receptor distribution and binding properties at the neuromuscular junction during aging. Int J Dev Neurosci 8:629642. doi:10.1016/0736-5748(90)90058-A

13. Ryan MC, Christiano AM, Engvall E, Wewer UM, Miner JH, Sanes JR, Burgeson RE (1996) The functions of laminins: lessons from in vivo studies. Matrix Biol 15:369-381. doi:10.1016/ S0945-053X(96)90157-2

14. Hirata M, Sakuma K, Okajima S, Fujiwara H, Inashima S, Yasuhara M, Kubo T (2007) Increased expression of neuregulin-1 in differentiating muscle satellite cells and in motoneurons during muscle regeneration. Acta Neuropathol 113:451-459. doi: 10.1007/s00401-007-0198-5

15. Andersson AM, Olsen M, Zhernosekov D, Gaardsvoll H, Krog L, Linnemann D, Bock E (1993) Age-related changes in expression of the neural cell adhesion molecule in skeletal muscle: a 
comparative study of newborn, adult and aged rats. Biochemical J 290:641-648

16. Bambrick L, Gordon T (1994) Neurotoxins in the study of neural regulation of membrane proteins in skeletal muscle. J Pharmacol Toxicol Methods 32:129-138. doi:10.1016/1056-8719(94) 90066-3

17. Prakash YS, Miyata H, Zhan WZ, Sieck GC (1999) Inactivityinduced remodeling of neuromuscular junctions in rat diaphragmatic muscle. Muscle Nerve 22:307-319. doi:10.1002/ (SICI) 1097-4598(199903)22:3<307::AID-MUS3>3.0.CO;2-M

18. Sieck GC, Fournier M (1989) Diaphragm motor unit recruitment during ventilatory and nonventilatory behaviors. J Appl Physiol 66:2539-2545

19. Prakash YS, Miyata H, Zhan WZ, Sieck GC (1995) Adaptations of diaphragm neuromuscular junction following inactivity. Acta Anat 154:147-161. doi:10.1159/000147762 